\title{
Study on Gels of Poly(methyl methacrylate) Stereocomplex by Light Scattering
}

\author{
Issa A. KATIME and José R. QUINTANA \\ Departmento de Quimica Fisica, Grupo de Polimeros, \\ Facultad de Ciencias, Universidad del País Vasco, \\ Bilbao 644, Bilbao, España
}

(Received November 16, 1987)

\begin{abstract}
We examined the formation and stability of poly(methyl methacrylate) (PMMA) gels. The gels were formed by mixing semidilute solutions of iso and syndiotactic PMMA in dimethyl formamide (DMF) at different temperatures. We studied the influences of polymer concentration, stereoregularity of the syndiotactic component and temperature in the kinetics of gel formation, and in the thermal stability of the gels formed. The analysis of this phenomenon has been carried out by light scattering. We verified the existence of a necessary minimum length of tactic sequences for these sequences to associate. This length increases with temperature. We also observed the existence of a bounding concentration from which the interactions, produced between tactic chains, pervade the whole solution forming a stiff gel. Below this concentration, the stereocomplex can also form perceptible aggregates but without involving the whole solution even after a long time of complexation.
\end{abstract}

KEY WORDS Gels / Poly(methyl methacrylate) Stereocomplex / Aggregation Process / Complexing Solvent / Radius of Gyration / Intermolecular Association / Stereoassociation /

The mixing of solutions of syndiotactic and isotactic poly(methyl methacrylate) (iso- and syn-PMMA) in suitable solvents leads to the formation of a stereocomplex by intermolecular association of isotactic and syndiotactic sequences. ${ }^{1-10}$

Very concentrated solutions of isotactic and syndiotactic PMMA form stiff gels ${ }^{1-4}$ which melt sharply, suggesting that extensive aggregation leads gradually to gelation. Such behaviour is typical of macromolecular systems cross-linked to form small crystallites, such as gelatine.

According to Watanabe et al., ${ }^{1}$ these gels are only slightly affected by changes in polymer concentration from about 1 to $9 \%$ by wt or by changes in the ratio of isotactic to syndiotactic polymer from about $1 / 4$ to $4 / 1$ by wt, only slightly affected by the molecular weight if $\bar{M}_{v}>100000$ and markedly dependent on the tacticity of the polymer.

In this paper, the formation of stereogels and thermal stability of the formed gels are examined. This behaviour was analyzed at different polymer total concentrations, tacticities of the components and temperatures of gel formation.

In order to know the influence of the tacticity we used two different pairs of polymers, mixing a same isotactic PMMA with two syndiotactic ones of different tacticity in the ratio of isotactic to syndiotactic $1 / 2(w / w)$.

We used dimethylformamide (DMF) as solvent. This liquid is considered a strongly complexing solvent and its high boiling point allows us to melt the gels by increasing temperature. The concentrations used were 1.00 and $2.00 \mathrm{~g} \mathrm{dl}^{-1}$.

As an experimental technique, we used light scattering, which allow us to easily follow the 
formation kinetics as well as the melting of the gels.

\section{EXPERIMENTAL}

Isotactic and syndiotactic PMMA samples (JR and SW) were supplied by Dr. W. Wunderlich, from Rohm GmbH Chemische Fabrik, Darmstadt, Germany.

A syndiotactic PMMA sample PO was prepared via free radical polymerization in benzene at $343 \mathrm{~K}$ using 1,2-azobisisohutyronitrile as initiator. ${ }^{11)}$

The weight average molecular weights, $\bar{M}_{w}$, were determined by light scattering, in ethyl acetate solutions at $298 \mathrm{~K}$ using a FICA 50 light scattering photometer. The GPC measurements were carried out in THF at $298 \mathrm{~K}$, using two $\mu$-Shodex columns (A80M) calibrated with polystyrene standards. The tacticities of the PMMA samples were determined from spectra measured on solutions in chloroform at $318 \mathrm{~K}$ by a $200 \mathrm{MHz}{ }^{1} \mathrm{H}-\mathrm{NMR}$ Brucker spectrometer. The molecular and structural parameters of the samples in this work are given in Table I. Sequence length distributions for both syndiotactic polymers can be taken from Figures 1 and 2, they were calculated applying first-order Markov statistics according to the method of Johnsen. ${ }^{12)}$

The gel formations were carried out in DMF solutions at the concentrations of 1.00 and $2.00 \mathrm{~g} \mathrm{dl}^{-1}$. Solutions of the PMMA were optically purified by centrifugation at $20000 \times g$ for a period of $3.00 \mathrm{~h}$. After centrifugation, the solutions were mixed directly into the light scattering cells, at the ratio of the isotactic to syndiotactic polymer $1 / 2(\mathrm{w} / \mathrm{w})$.

In order to get good mixture homogeneity, the mixture was heated to $363 \mathrm{~K}$ at which gel formation is not possible; it was then stirred and quenched to the temperature at which the gel would formed.

The kinetics of gel formation were followed by light scattering. We measured the scattered light intensity at eleven angles between 30 and
Table I. Weight average molecular weight, $\bar{M}_{w}$, polydispersity, $\bar{M}_{w} / \bar{M}_{n}$, and stereoregularity of the PMMA samples

\begin{tabular}{lcrrrr}
\hline $\begin{array}{c}\text { PMMA } \\
\text { sample }\end{array}$ & $\bar{M}_{w}$ & $\bar{M}_{w} / \bar{M}_{n}$ & $\% \mathbf{~ I}$ & $\% \mathrm{H}$ & $\% \mathrm{~S}$ \\
\hline JR & 400000 & 1.34 & 90 & 7 & 3 \\
SW & 165000 & 1.23 & 1 & 26 & 73 \\
PO & 150000 & 1.52 & 5 & 40 & 55 \\
\hline
\end{tabular}

I, H, S: content of iso-, hetero-, syndiotactic triads, respectively.

$350^{\circ}$ at various times during the gelation formation and analysed the evolution of the process. A FICA 50 light scattering photometer with vertically polarized incident light was used. We used light scattering cells which could be closed to avoid possible solvent loss.

The same instrument was used to follow the melting of the gels. Its thermostating system allowed us to attain a nearly constant heating rate of $1.5 \mathrm{~K} \mathrm{~min}^{-1}$.

Throughout the melting of the gels, the intensity scattered at an angle of $90^{\circ}$ was registered by a digital voltmeter and a two-pen recorder. The temperature could be measured by introducing a thermistor into the gel. This thermistor, previously calibrated with a Hewlett-Packard quartz 2801A thermometer, was put on a digital voltmeter and recorded.

\section{RESULTS AND DISCUSSION}

\section{Formation of Stereocomplex Gels}

The kinetics of gel formation has been followed by light scattering, analyzing the evolution of the scattered intensity throughout time, as a function of the observation angle. The kinetics were studied at 273, 298, and $323 \mathrm{~K}$ for both polymer pairs, JR/SW and JR/ PO.

First, we analyzed the gel formation in solution at a concentration of $2.00 \mathrm{~g} \mathrm{dl}^{-1}$. Figures 3 and 4 show two examples of the variation of the scattered intensity with time, given in a logarithmic scale, in the gelation of 
Gels of PMMA

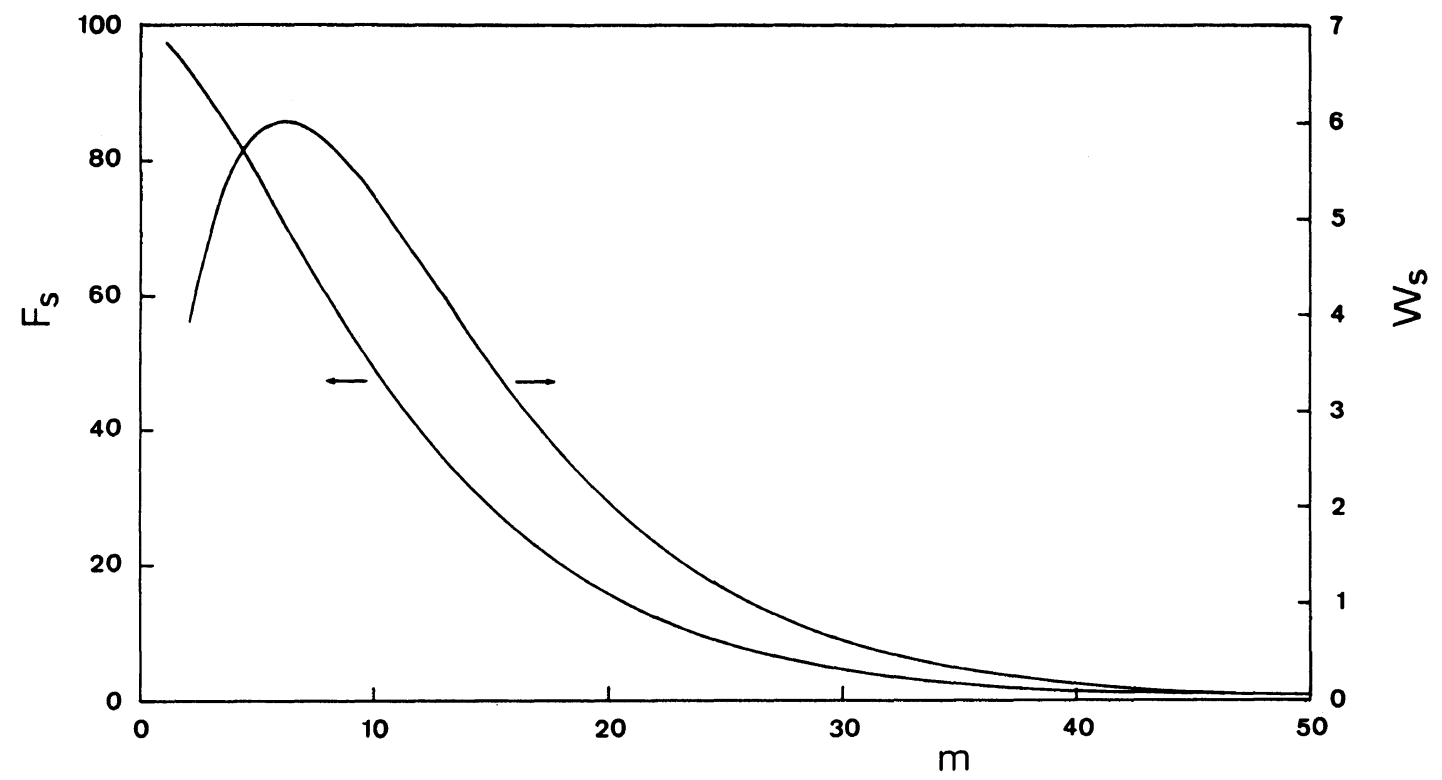

Figure 1. Integrated $\left(F_{\mathrm{s}}\right)$ and differential $\left(W_{\mathrm{s}}\right)$ weight distribution of syndiotactic sequences as a function of length in monomeric units, $m$, for PMMA sample SW.

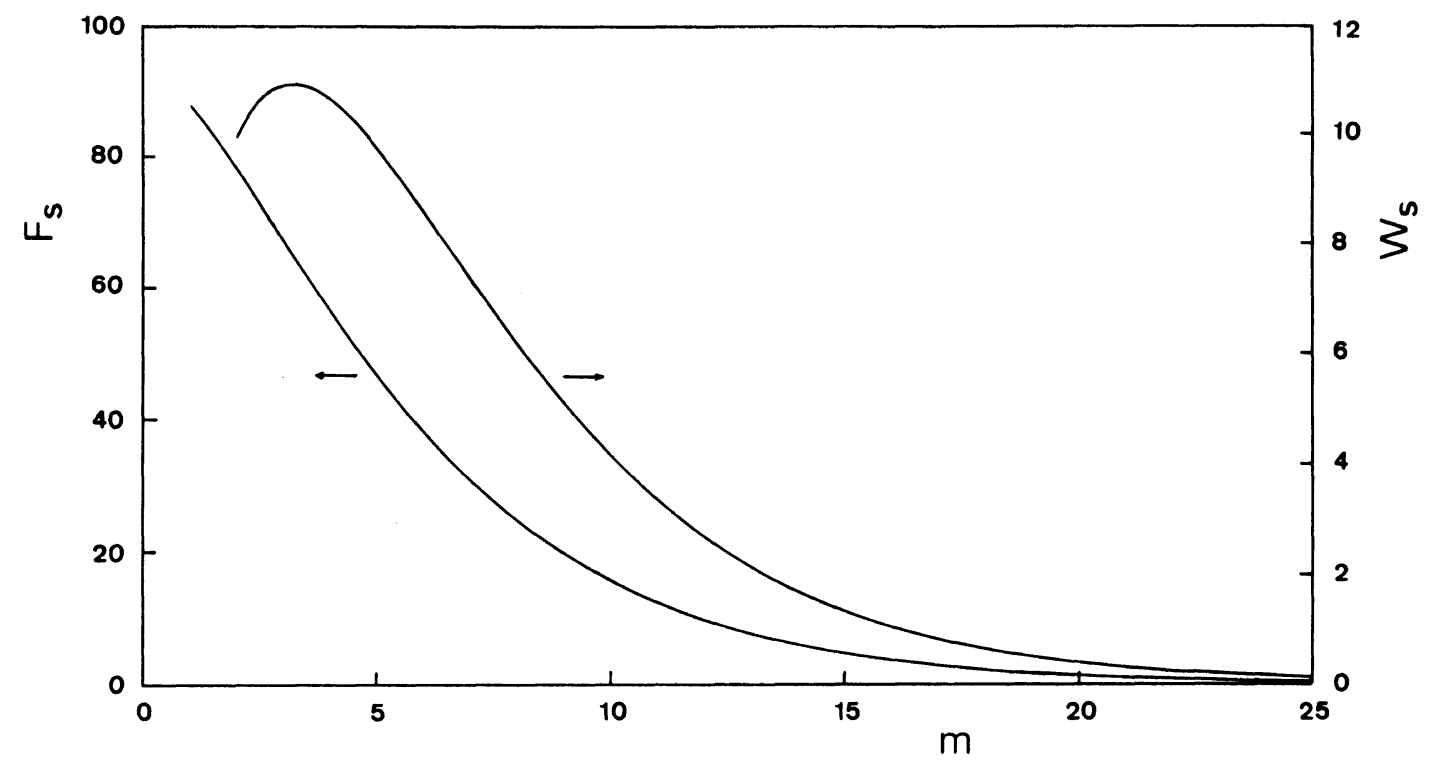

Figure 2. Integrated $\left(F_{\mathrm{s}}\right)$ and differential $\left(W_{\mathrm{s}}\right)$ weight distribution of syndiotactic sequences as a function of length in monomeric units, $m$, for PMMA sample PO.

Polymer J., Vol. 20, No. 6, 1988 


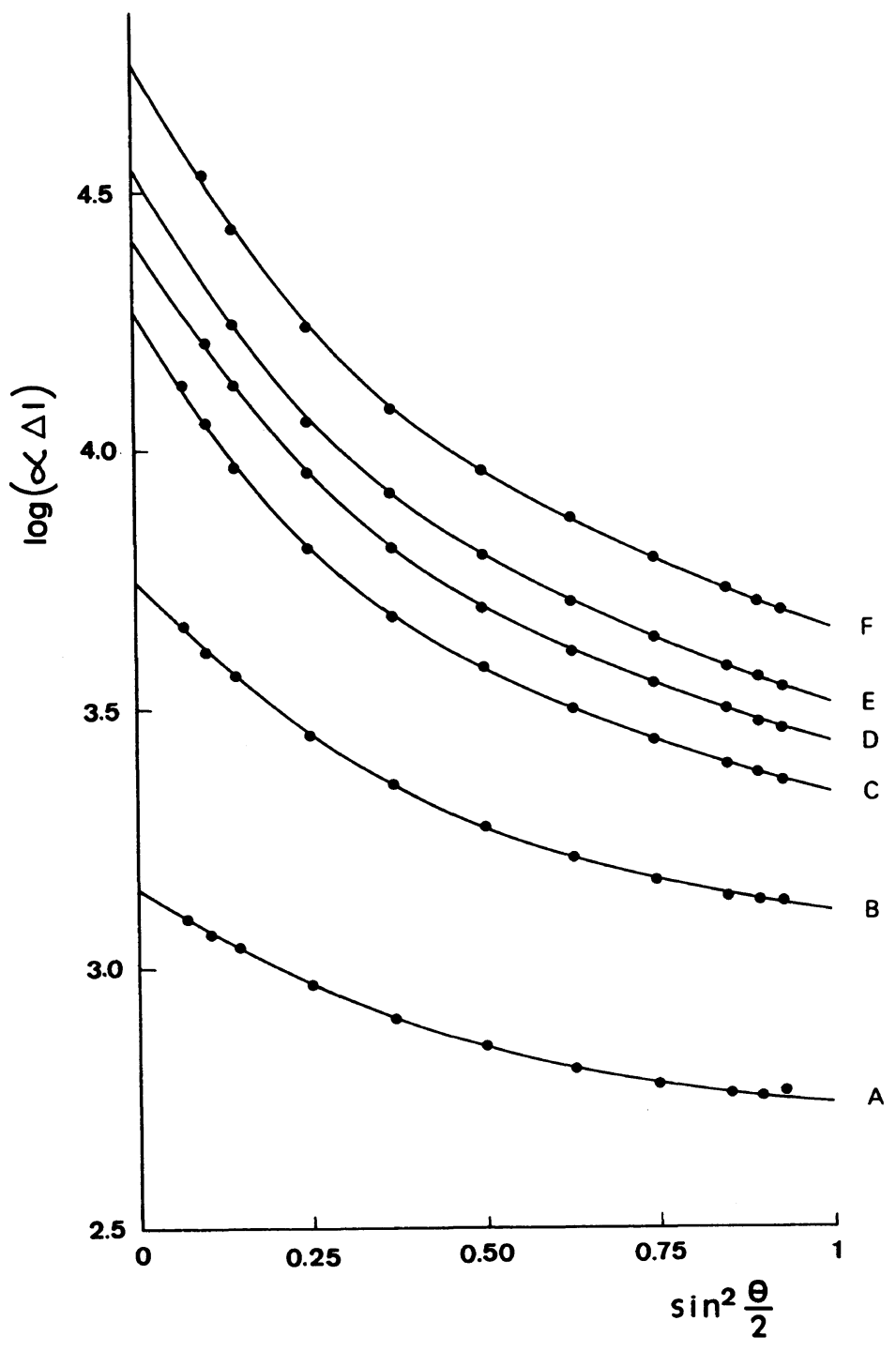

Figure 3. Evolution of scattered intensity with time as a function of the observation angle for the polymer pair JR/SW at the concentration $2.00 \mathrm{~g} \mathrm{dl}^{-1}$ and at $298 \mathrm{~K}$. A, 0.07; B, 0.17; C, 0.33; D, 0.67; E, 1.67 ; and F, $16.72 \mathrm{~h}$.

polymer pairs JR/SW and JR/PO, respectively, at $298 \mathrm{~K}$. We used the Guinier plot $^{14}$ because it is more representative than the ordinary $I^{-1}$ plot when the variations of the observed intensity $I$ are very high. As can be seen, the variation of $\log \alpha \Delta I$ with $\sin ^{2}(\theta / 2)$ is not linear; the scattering intensity is larger at small angles. This behaviour can be due to heterogeneity in the stereocomplex solution or gel.

A similar study was carried out at $298 \mathrm{~K}$ with solution of both polymer pairs at a concentration of $1 \mathrm{~g} \mathrm{dl}^{-1}$. The variations of the scattered intensity were similar to those obtained at $2.00 \mathrm{~g} \mathrm{dl}^{-1}$. However, there exists a remarkable difference: the stereocomplex ag- 


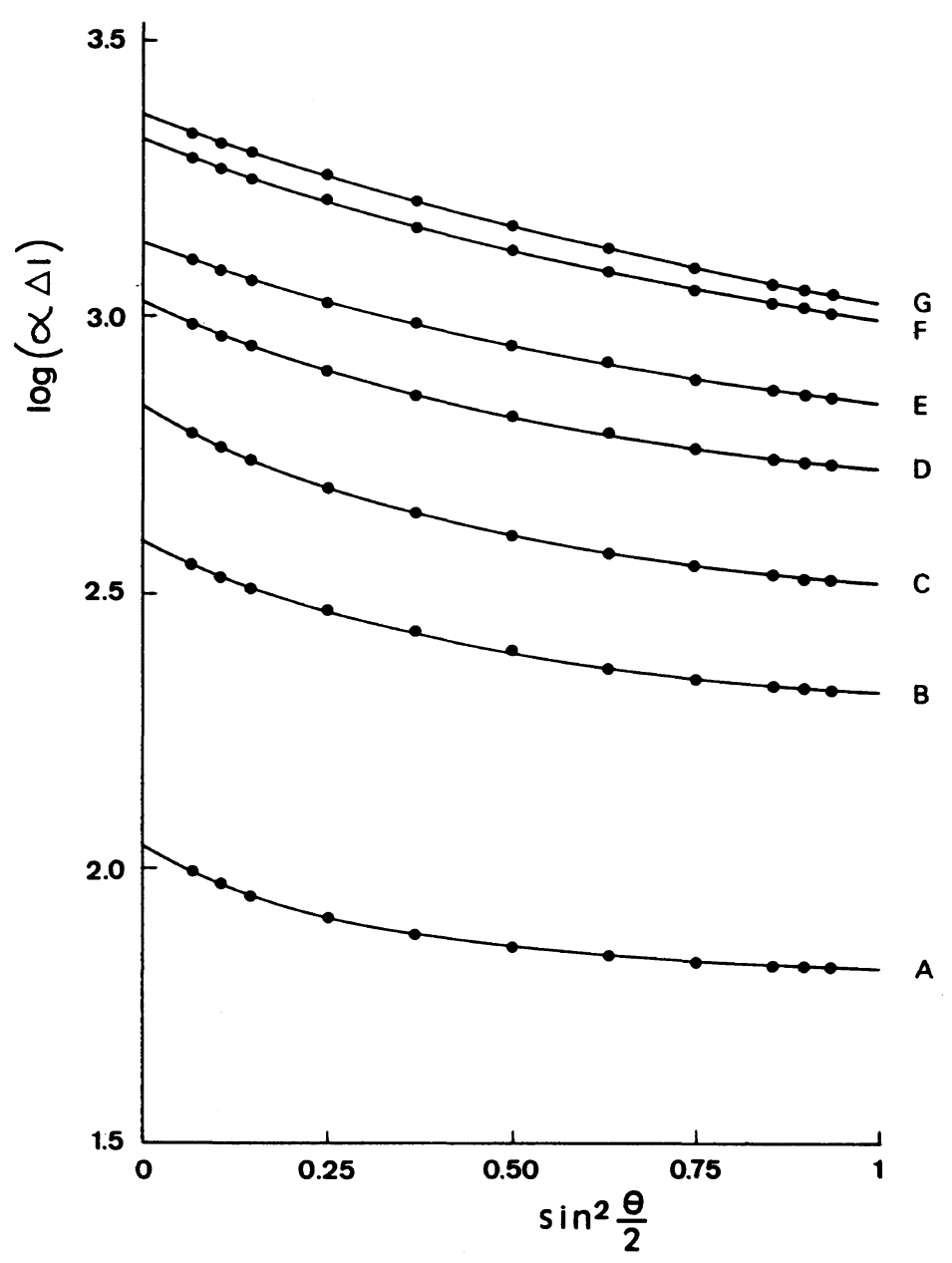

Figure 4. Evolution of scattered intensity with time as a function of the observation angle for the polymer pair JR/PO at the concentration $2.00 \mathrm{~g} \mathrm{dl}^{-1}$ and at $298 \mathrm{~K} . \mathrm{A}, 0 ; \mathrm{B}, 0.13 ; \mathrm{C}, 0.32 ; \mathrm{D}, 1.43 ; \mathrm{E}, 4.70$; F, 24.52; and $G, 47.35 h$.

gregates at $c=1 \mathrm{gdl}^{-1}$ did not prevade the whole solution to form a stiff gel, even after a long time.

Figures $5-7$ summarize the obtained results as a function of the experimental conditions: tacticity of syndiotactic PMMA, polymer total concentration and temperature. In order to describe the evolution of the scattered light with time, we considered characteristic magnitudes: the scattered intensity extrapolated to zero angle: $\left.\log (\alpha I)\right|_{\boldsymbol{\theta \rightarrow 0}}$ and the dissymmetry given by

$$
Z=(\Delta I)_{45} /(\Delta I)_{135^{\circ}}
$$

where $\alpha$ is the angle correction and $\Delta I$ is the excess light scattering intensity.

Figure 5 shows the general form of the time dependence of gel formation $\left(c=2.00 \mathrm{~g} \mathrm{dl}^{-1}\right)$ and the influence of the temperature and tacticity. For any temperature, the formation of the gel is fast and reaches a stability after 10 or $20 \mathrm{~h}$. In fact, it is rather a pseudo-stability because there is always a very slow evolution of the gel and, sometimes, it releases solvent. 


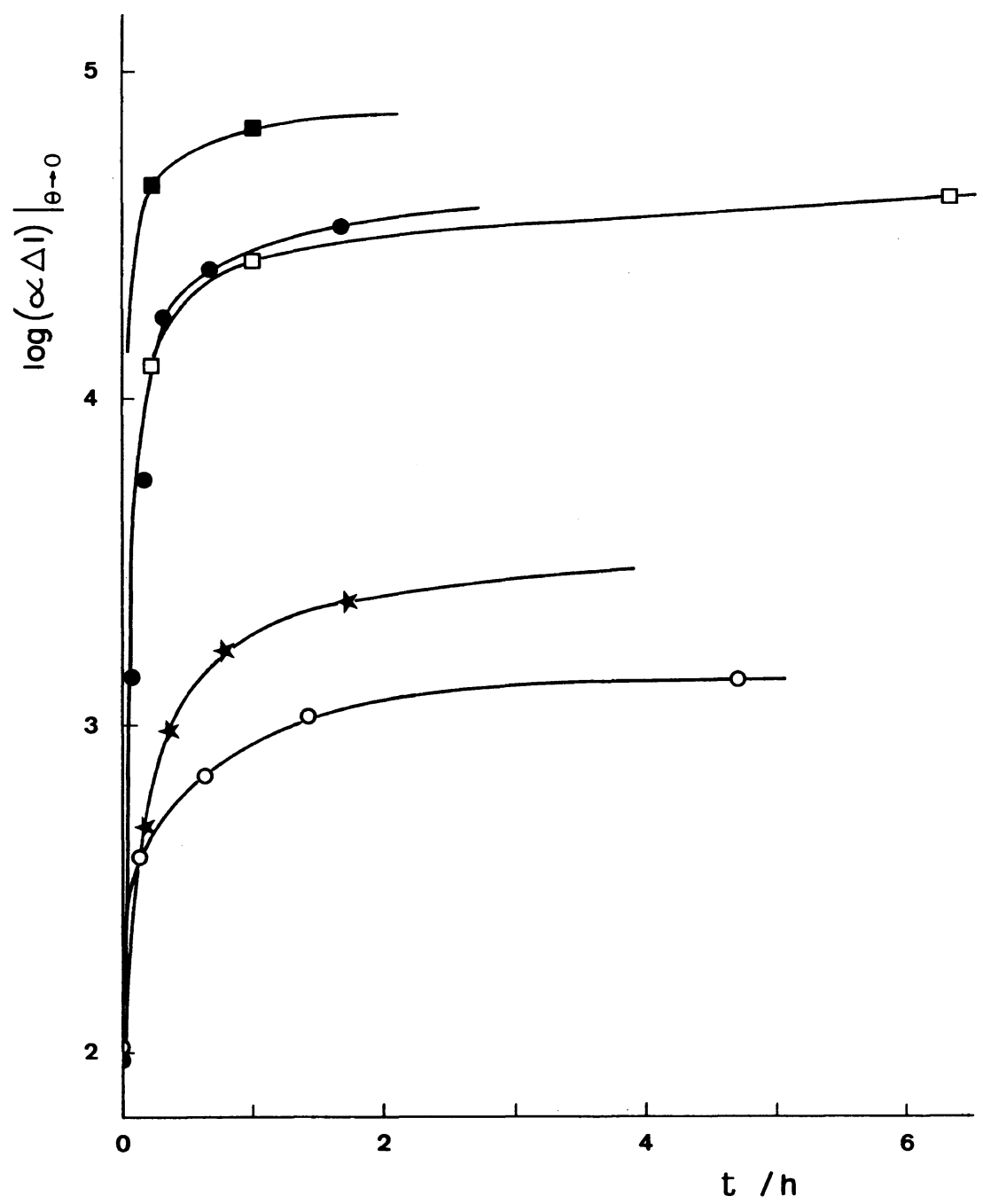

Figure 5. Time dependence of the scattered intensity extrapolated to zero angle, as a function of tacticity and temperature of gel formation, at a concentration $2.00 \mathrm{~g} \mathrm{dl}^{-1}$. JR/SW at $273(\mathbf{\square}), 298(\mathbf{O})$, and $323 \mathrm{C}$ $(\star) ; J R / P O$ at $273(\square)$ and $298 \mathrm{~K}(\mathrm{O})$.

As we can see, for a decrease in the tacticity of the polymeric components, there is a decrease in the rate of gel formation and in the scattered light intensity levels. A decrease of the temperature has an opposite effect.

Figure 6 shows the influence of the polymer total concentration and tacticity. As we can see, the influence of concentration on the evolution of the scattered light is very smaller than the influence of the tacticity or tempera- ture, as was shown by Watanabe et al. ${ }^{1}$

We found that the influence of the tacticity on the stereocomplex formation depends markedly on temperature. In Figure 5 it can be observed that at $273 \mathrm{~K}$, the difference between both polymer pairs is smaller than at $298 \mathrm{~K}$. This can be explained if we consider the existance of a necessary minimum length sequence to form crystallites. As the temperature decreases this minimum sequence 


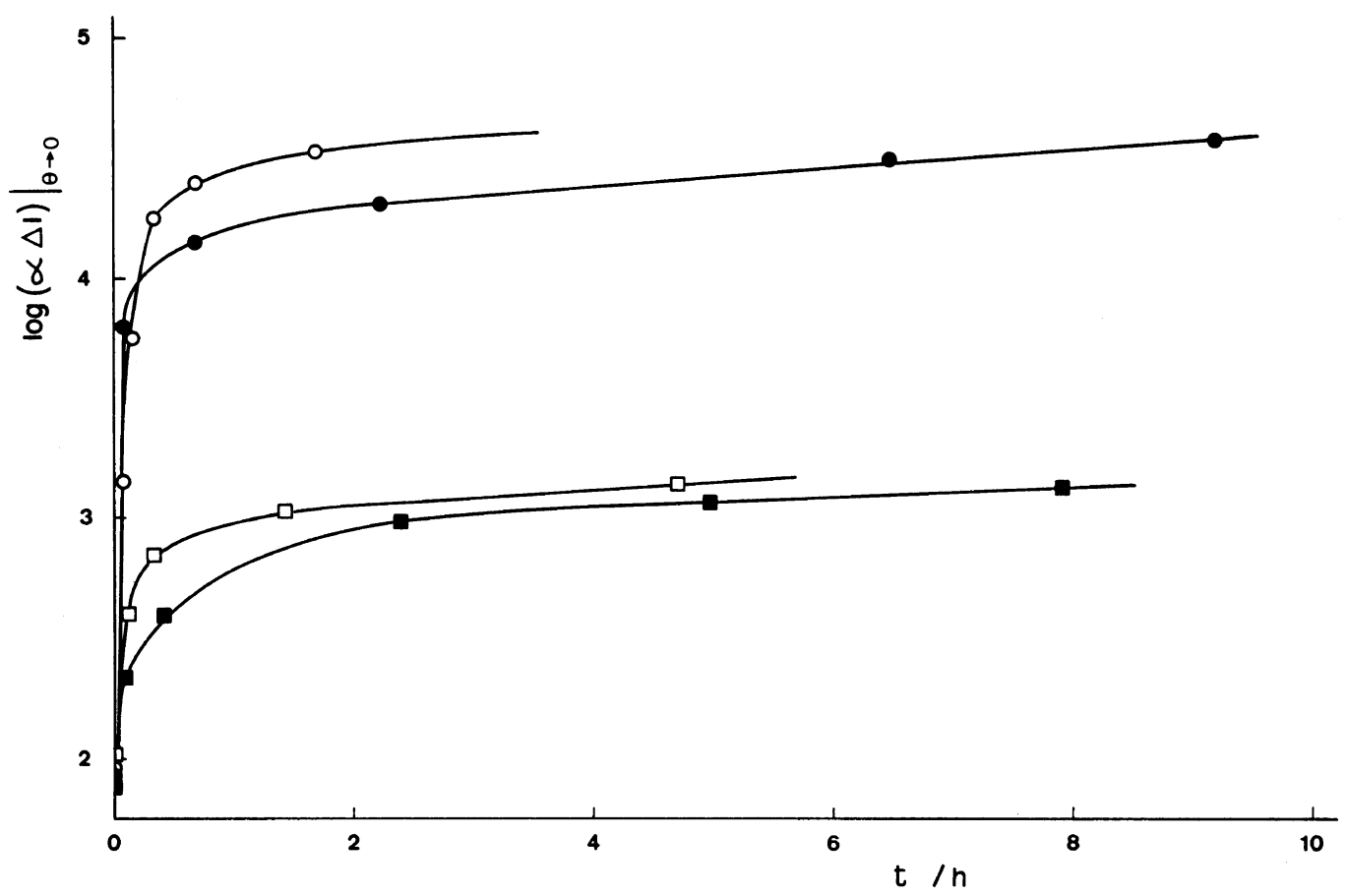

Figure 6. Time dependence of the scattered intensity extrapolated to zero angle, as a function of tacticity and concentration at $298 \mathrm{~K}$. JR/SW, $1(\bigcirc)$ and $2.00 \mathrm{~g} \mathrm{dl}^{-1}(\bigcirc) ; \mathrm{JR} / \mathrm{PO}, 1(\square)$ and $2.00 \mathrm{~g} \mathrm{dl}^{-1}(\square)$.

length decreases.

The scattered light intensity due to gelation is very strong and has high dissymmetry coefficients. For dilute or semi-dilute polymer solutions, as well as for homogeneous or ideal gels, the scattered light due to concentration (or segment density) fluctuations is weak, as seen at the initial stage of gelation or after the melting of the gels.

In our case of a concentration $1.00 \mathrm{~g} \mathrm{dl}^{-1}$, the scattered light of the gels was $100-400$ times stronger than that of the solutions. This large increase in scattering can be only explained by significant heterogeneities which originate during gel formation.

In Figure 7 a polymer network is schematically shown. There exist zones with a high concentration of segments (zones 1 and 3 ) and that with a lower segment density (zone 2).

The evolution of dissymmetry with time at different tacticities and concentrations is plot-

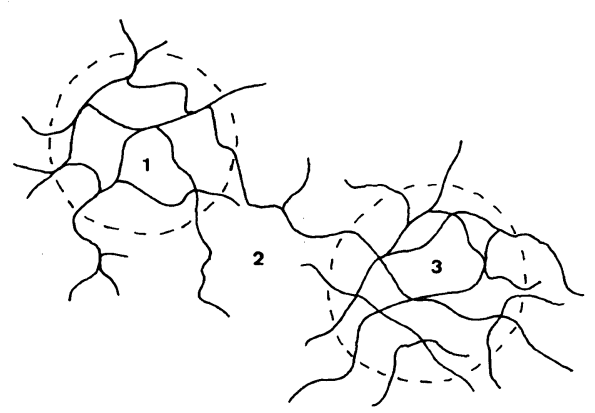

Figure 7. Schematic representation of the structure of a polymer network.

ted in Figure 8. The dissymmetry can be related to the size of heterogeneities in the gel. As we can see in this figure, an increase in the tacticity leads to an increase in the dissymmetry, suggesting that the heterogeneity of the system occurs in a considerably large spatial scale. This fact seems reasonable because, as it can be seen in Figure 1, the syndiotactic 


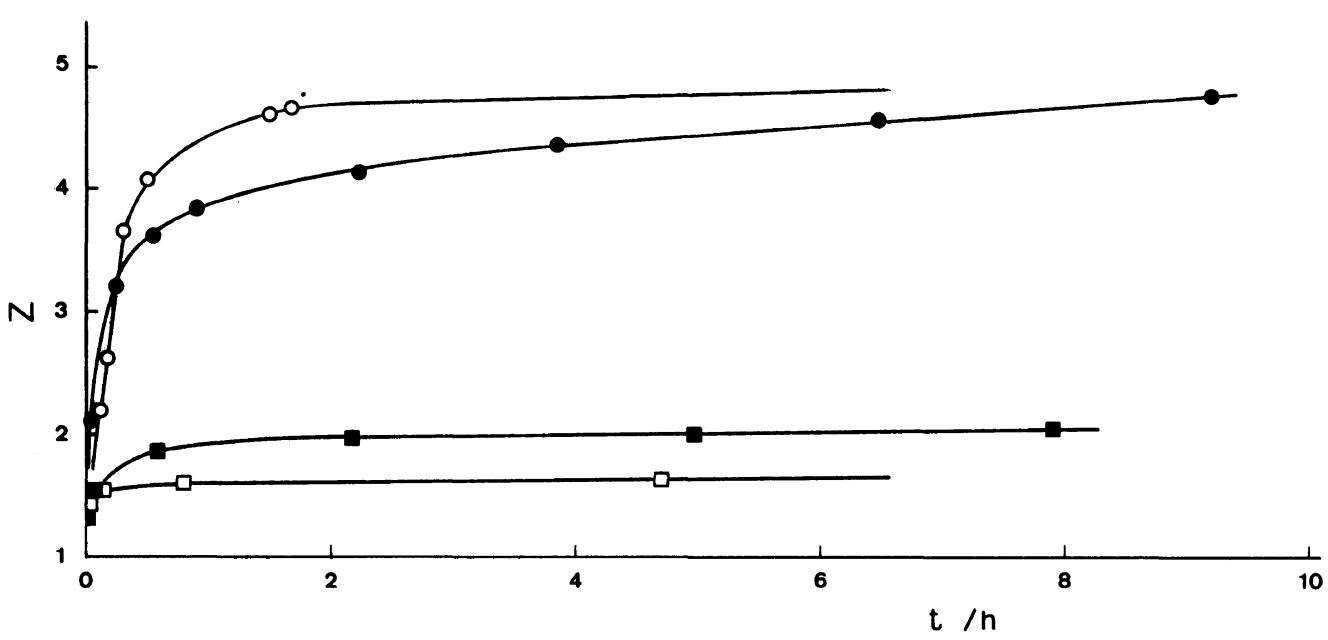

Figure 8. Time dependence of the dissymmetry as a function of the tacticity and the concentration at $298 \mathrm{~K} . \mathrm{JR} / \mathrm{SW}, 1$ (O) and $2.00 \mathrm{~g} \mathrm{dl}^{-1}(\mathrm{O})$; JR/PO, $1(\boldsymbol{\square})$ and $2.00 \mathrm{~g} \mathrm{dl}^{-1}(\square)$.

PMMA SW has longer syndiotactic sequences which form bigger crystallites.

\section{Melting of Stereocomplex Gels}

A study on the thermal stability of the gels $\left(c=2.00 \mathrm{~g} \mathrm{dl}^{-1}\right)$ was also carried out. In Figure 9 , the variation of the scattered light at $90^{\circ}$ during the melting of the JR/PO gels formed at 273 and $298 \mathrm{~K}$ is shown. The melting of the JR/ SW gels formed at 273,298 , and $323 \mathrm{~K}$ is shown in Figures 10 and 11, respectively.

On comparing the melting curves as a function of the tacticity of the syndiotactic PMMA, several differences can be observed. First, there exists a great difference in the temperature at which melting finishes, the highest one being for the pair JR/SW. This can be explained because the polymer $\mathrm{SW}$ has longer syndiotactic sequences which form stronger links; therefore the melting temperature increases. As regards the temperature at which melting begins, the difference is smaller, since the pair JR/SW also forms weak links.

This pair also shows a larger melting range. This can be due to the distribution of sequences of the syndiotactic PMMA. As we can see in Figures 1 and 2, PMMA SW has a broader molecular weight distribution than that of PMMA PO.

Another interesting factor in the gel formation is temperature. This influence is displayed, in the melting curves of the formed gels as well as in the kinetics. We can see that the beginning of the melting shifts to higher temperatures as the temperature of gel formation increases. This supports the hypothesis of the existence of a minimum length of stereoregular sequences necessary for stereocomplex formation. ${ }^{7,16}$ Thus, as the temperature of the gel formation increases, the minimum length increases and in this way the weakest links are hindered. There also exist small differences between the temperatures at which the melting finishes; the higher the gel formation temperature is, the higher that temperature is. This increment can be due to the steric hindrances that is, as the gel formation temperature increases, the number of links between chains decreases for a given time of gel formation. In this way a larger mobility of the chains is allowed and therefore the links can become more extensive.

We can also observe that as the gel for- 


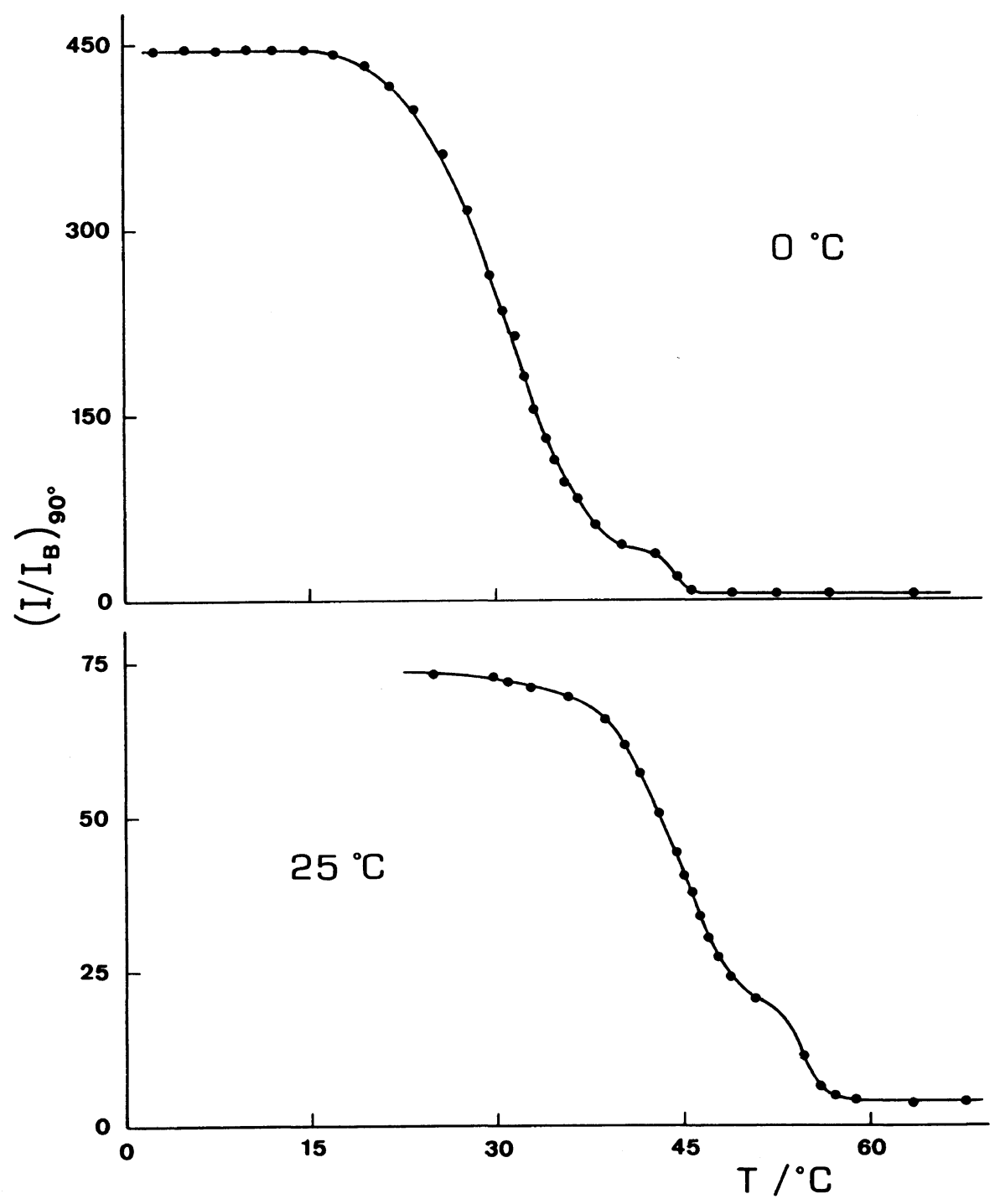

Figure 9. Variation of the scattered intensity during melting of the gels formed at 273 and $298 \mathrm{~K}$, corresponding to the polymer pair JR/PO.

mation temperature decreases, the melting range gets broad. This may be because the lower the gel formation temperature is, the shorter is the minimum length of stereoregular sequences necessary for associating and in consequence, there will exist a larger diversity of links as regards stability.

As mentioned above, the pair JR/PO does not form a gel at $323 \mathrm{~K}$. This makes us think that the sample PO does not have enough syndiotactic sequences with minimum length necessary to form stable links at that temperature.

Acknowledgments. The authors thank Dr. Claude Strazielle of the Institute Charles Sadron (CRM-EAHP) his helpful support.

We are indebted to Vicerrectorado de 


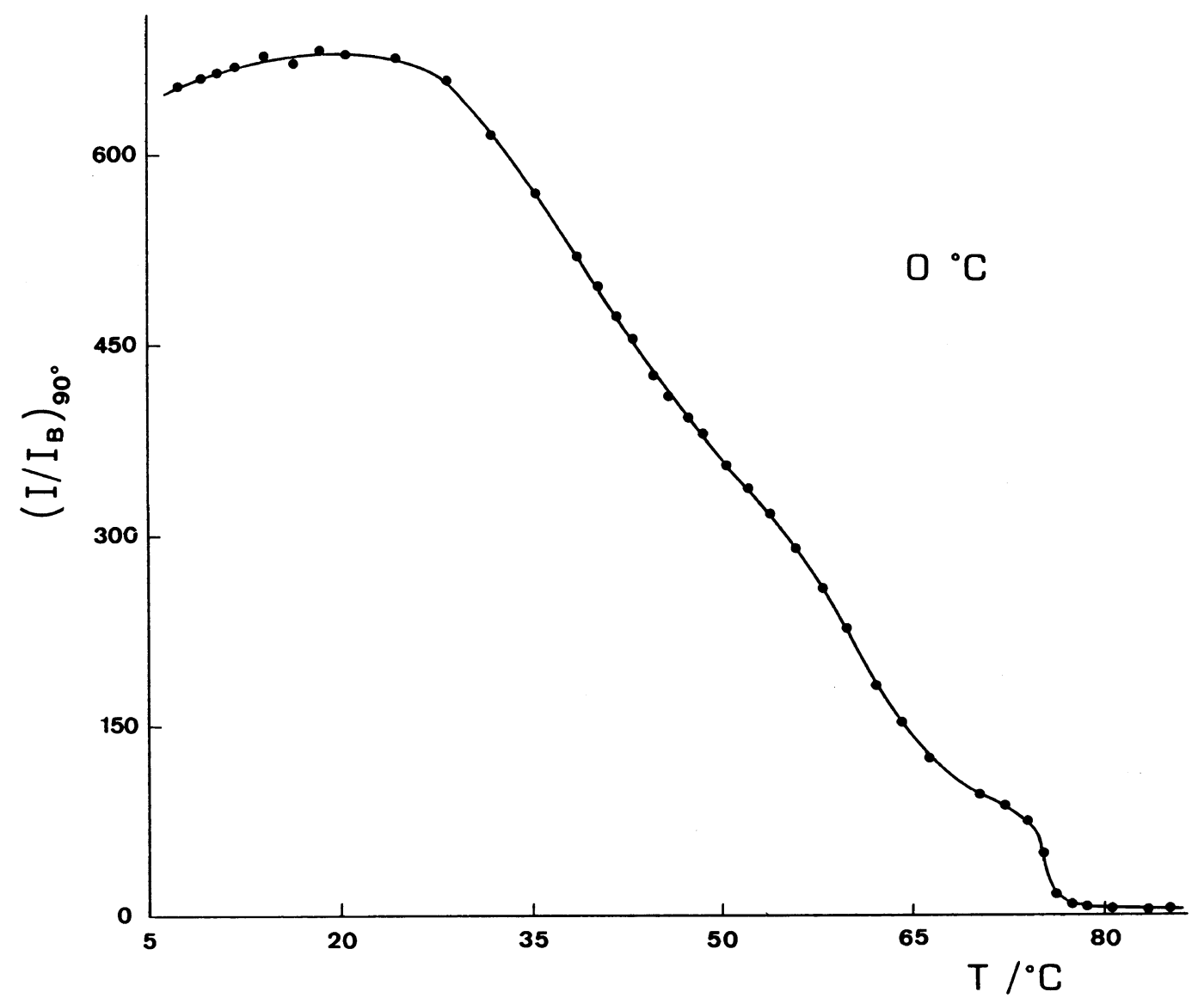

Figure 10. Variation of the scattered intensity during melting of the gel formed at $273 \mathrm{~K}$ corresponding to the polymer pair JR/SW.

Investigación de la Universidad del Pais Vasco for financial support.

\section{REFERENCES}

1. H. Watanabe, C. F. Ryan, P. C. Fleisher, and B. S. Garrett, J. Phys. Chem., 65, 896 (1961).

2. C. F. Ryan and P. C. Fleisher, Jr., J. Phys. Chem., 69, 3384 (1965).

3. A. M. Liquori, G. Anzuino, V. M. Corio, M. d'Alagni, P. de Santis, and M. Savino, Nature, 206, 358 (1965).

4. W. Borchard, M. Pyrlik, and G. Rehage, Makromol. Chem., 145, 169 (1971).

5. H. Z. Liu and K. J. Liu, Macromolecules, 1, 157 (1968).
6. N. G. Belnikevitch, L. Mrkvickova, and O. Quadrat, Polymer, 24, 713 (1983).

7. J. Spevacek and B. Scheneider, Makromol. Chem., 715, 2939 (1974).

8. I. Katime, J. R. Quintana, and J. Veguillas, Polymer, 24, 903 (1983).

9. J. R. Quintana and I. Katime, J. Chem. Soc., Faraday Trans. 1, 82, 1333 (1986).

10. I. Katime, J. R. Quintana, and J. Veguillas, Eur. Polym. J., 21, 1075 (1985).

11. I. Katime, "Química Física Macromolecular," Editorial Del Castillo, Madrid, 1979.

12. U. Johnsen, Kolloid Z.-Z. Polym., 178, 161 (1961).

13. M. Duval and H. Coles, Rev. Phys. Appl., 15, 1399 (1980).

14. M. B. Huglin, Ed., "Light Scattering from Polymer Solutions," Academic Press Inc., London, 1972. 


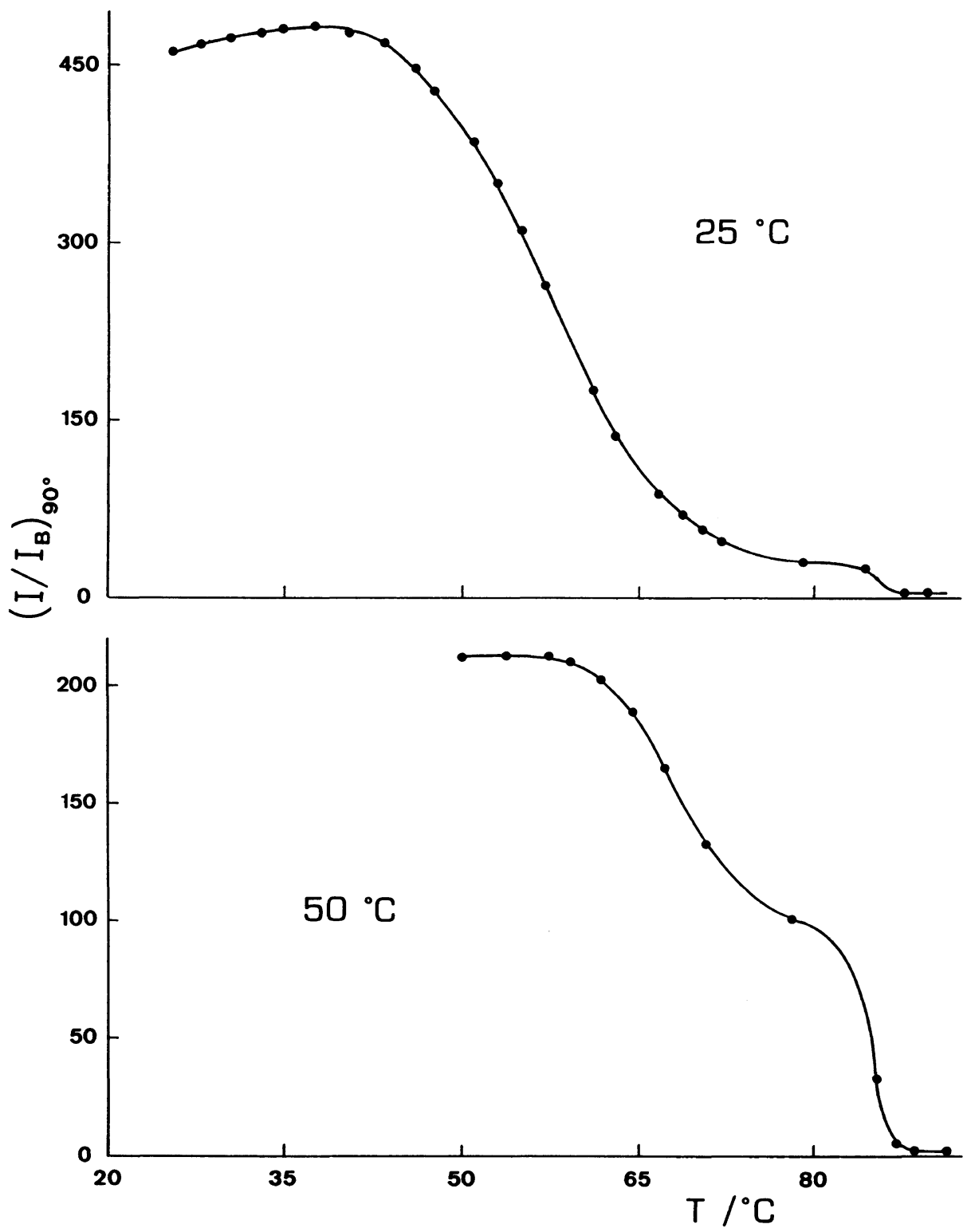

Figure 11. Variation of the scattered intensity during melting of the gels formed at 298 and $323 \mathrm{~K}$ corresponding to the polymer pair JR/SW.

15. P. Debye and A. M. Bueche, J. Appl. Phys., 20, 518 (1949).
16. I. Katime, J. R. Quintana, and C. Strazielle, Makromol. Chem., 187, 1441 (1986). 